\title{
Comprehensive Detection Technology for Urban Subway
}

\author{
Jinghui Xia ${ }^{1}$, Tao Liao ${ }^{2}$ and Lixun Zhang ${ }^{1}$ \\ ${ }^{1}$ Zhengzhou Rail Transit Co., Ltd., China \\ 2 State Grid Henan Electric Power Research Institute, Zhengzhou, China
}

\begin{abstract}
The urban subway comprehensive detection system is a complex system, which is the collection of advanced sensor technology, computer technology, network technology, data analysis, image analysis and processing technology, artificial intelligence technology and so on. The bow net, orbit and threshold detection of basic project and detection principle for subway are expounded in this paper. And the composition and development of subway comprehensive detection system is proposed.
\end{abstract}

\section{Introduction}

At present, the rapid development scale and speed of China's urban subway are unprecedented. Testing the bow net, orbit and tunnel limits, and then timely discovering and dealing with potential safety hazard are the necessary measures to ensure the safe operation of the subway [1]

Urban rail transit includes three types of transportation: the subway, overhead, and light rail. The subway is in underground, do not take up floor space, plus special orbit, so compared with other ways of urban rail transit, the subway has great superiority. For example, the subway has quick speed, not traffic jam, safe, comfortable, and the advantages of small disturbance.

The function of bow net, orbit and threshold detection for electric railway integrated detection technology has been widely used in China's high-speed rail and conventional electrified railway. In the early time, on the basis of introduction of foreign technology, integrated checkout automobile has developed which is suitable for high-speed rail and electric rail.

Compared with the high speed railway and electric rail, urban subway has its characteristics in running speed, line feature parameters, tunnel length, power supply system, operation management and so on. It is not realistic that using the integrated checkout automobile for high-speed rail and electric rail simply in the urban subway. It will Faced with the problems of adaptability, cost and so on.

It is quite necessary and urgent to analysis and research the integrated detection technology that is suitable for urban subway, to research, develop and improve urban subway integrated checkout automobile [2], [3].

\section{Dynamic detection of bow net}

(1) The main detection project
Bow net is adopted widely in metro vehicle a current device, belonging to one of the key components of the train and its status directly affect the safe operation. Traditional inspection methods rely on artificial Govinda check, need to be done after a special power of the same inside the car depot. Train pantograph online monitoring system by using high speed image processor and sensors, with image analysis method, on the premise of not affect normal operation of the train to realize online detection of pantograph. Detection efficiency is high, the system can real-time tracking monitoring various pantograph mainly, the feature information of the running state and safety performance, and comprehensive analysis of the information processing, timely forecast affecting the safe operation of the vehicle pantograph fault, eliminate the problems.

Bow net [4] system is composed of contact system and pantograph. The main project for dynamic detection of bow net include: contact wire height (Including rigid suspension and flexible suspension), stagger value, double contact line horizontal distance and height difference, anchor segment joint contact line horizontal distance and height difference, the high change rate of high conductivity, contact pressure of bow net, offline of bow net, impact of bow net, network voltage and so on. Bow net system dynamic detection should be implemented whether in the neutral and live cases.

(2) The main project of detection principle

1) Detection of stagger value and high conductivity

The parameter of stagger value, high conductivity and so on is detected using laser scanning sensor. Laser scanning sensor is an optical measurement system which is non-contact. It installs on the roof. The way of continuous emission of laser beam is used to measure the background or the object that is under test to get their two-dimensional planar.

$$
S=h / \tan \theta+C
$$




$$
H=h+h_{0}
$$

In the equation: $S$-stagger value; $H$ - conductor height; $h$-measurement height; $C$ - Deflected center distance of laser scanning sensor(left is negative, right is positive); $\theta-$ angle; $h_{0}$ - height of laser scanning sensor of the rail surface.

2) Detection of bow net contact pressure

Contact wire's layout is like the Chinese character “之”. The point application of contact force moves around within the scope of work in the skateboard. It is not only the position of the action of forces randomly changes, but also the direction of the contact force is changing. Contact force detection device should be able to run in the charge of cases and measure the contact force of wire in the vertical direction of the skateboard that is at any position [4], [5].

Its formula is shown in formula (3):

$$
F_{J}=F_{1}+F_{2}+F_{3}+F_{4} \pm m a-W
$$

In the equation: $F_{J}$-contact force of bow net in the vertical direction; $m$ - equivalent quality of the skateboard; $W$ - the weight of slide; $a$ - pantograph acceleration that is measured by accelerometer installed at the bottom of the pantograph; $F_{1}, F_{2}, F_{3}, F_{4}$ - the measured value of the force sensor that installed in the four corners of the bow head slide.

3) The detection of hard point

Hard point detection principle diagram is shown in Fig. 1. Pantograph formed the measured voltage by the sensor (the accelerometer) through the impact acceleration of the catenary hard point. The voltage is converted to frequency signal and then it will be transferred to optical communication channel by cable transmission. Optical communication channel can achieve reliable isolation between the high and low pressure. Low voltage signal is sent to the computer realize the hard point judge.

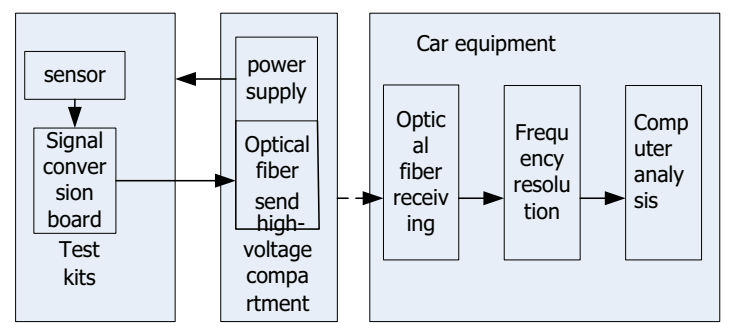

Figure 1. Schematic diagram of hard point detection

4) The detection of network voltage

Network voltage will be isolated by isolation transformation link. Then it will be converted to low voltage $(0-5 \mathrm{~V})$ and import to acquisition channel. The network voltage measurement will be realized.

5) The dynamic compensation

Two groups of laser displacement sensor will be installed on the vehicle bogie to measure horizontal displacement of the vehicle. Two sets of laser displacement sensor and damper are installed between car body and the bogie in order to measure the relative longitudinal displacement measurement of car body and the bogie. Based on two kinds of displacement measurements, dynamic compensation of position detection quantity in level and side will be got.

Dynamic detection of track

(1) The main detection project

The main project of dynamic detection of track include: the orbit of the left and the right of discretion, the orbit of the left and the right of direction, horizontal (superelevation), warp (twist warp), track spacing and track spacing rate, curvature, vertical and lateral acceleration of car body, the top of rail, flank wear and so on.

(2) The main principle of project detection

1) Track spacing and track spacing rate

Orbital shape and the distance between the two sensors can be measured by the two-dimensional laser outline sensor which is installed on the detection beam. Then it can get the horizontal distance between the right rail and left rail which is about $16 \mathrm{~mm}$ under the rail top. That is the track spacing value. And then track spacing rate can be obtained.

2) The top rail and flank wear

Pattern matching and comparing will be implemented between orbital shape measured by the two-dimensional laser outline sensor and orbital shape that was set in order to get sanding top and sanding side.

3) Track curvature

Angle of rotation that corresponded to the curve track can be measured by shaking gyroscope. Then chord length of the curve track can be obtained. The ratio of the chord length and the corresponding rotation Angle is the curvature.

4) Horizontal (superelevation)

Height difference within the same cross-sectional of two rail on the top surface is called horizontal (superelevation). Its detection principle: Absolute relative angle between beam and the earth can be measured by processing measurement signal that through inclinometer and roll gyroscope. The distance of beam and rail end face can be measured by the two-dimensional laser contour sensor. And then we can get the angle between rail and beam. Though the difference of the two angles between the top of the rail and the earth we can get the absolute angle. Absolute level high value can be obtained by conversion. Then through the transformation and minus between base value and the design, we can get relative horizontal value.

5) The triangle pit

Triangle pit refers to maximum deviation $(h)$ of the right and left orbit in top rail which is based on a chord length apart $L$. The basic general length is 2.2 meter.

6) Lateral acceleration and the vertical acceleration of car body

It can be measured through lateral acceleration sensor and vertical acceleration sensor installed in the car body. The measurement values can be get by removing the lateral acceleration of the centrifugal force and the initial base value of vertical acceleration.

Because of space, we escape the detection principle of orbital altitude between right rail and left rail.

7) Parts missing detection of bow net

On-line detection system using separate laser position sensor is used to identify the targeted, in order to judge 
whether the electric GongDuan Angle parts missing. As the train went by in the catenary at the top of the two laser position sensors for the electric GongDuan Angle position for continuous acquisition scan, will receive the reflected signal for data analysis, and judgment by electric GongDuan Angle part is complete.

After car, industrial computer through the network to read the image from the image sensor, and clear the cache initialization, such as to wait for the next shot. System reads the images after start to carry on the analysis, abrasion of pantograph slider carbon, carbon slider fracture defect, offset, tilt and missing parts such as state is analyzed, and returns the result.

\section{Threshold dynamic detection, Catenary inspection technology and integrated system}

\section{1) The high-speed railway catenary system}

Catenary is along the railway lines over the erection of transmission lines, is a special form of electric locomotive power supply. By the foundation and pillar, positioning device, supporting device with several contact suspension parts. Contact suspension including hanging string, the contact line, the carrier cable and connection parts. Contact suspension by supporting devices set up on the pillar, its function is to get electricity from traction substation to electric locomotive. Support device used to support contact suspension, and the load to the pillar or other buildings. According to the catenary's interval, yard and large buildings. Support device including suspension insulator string, horizontal bar, wrist, arm, rod insulator and other buildings of special support equipment. Positioning device includes locating pipe and locator, its function is fixed the position of the contact line, the line of contact within the scope of the pantograph slide running track, guarantee the contact line and pantograph not from, and will be transmitted to the level of the line of contact load. Catenary of task is to convey electricity directly from the traction substation for electric locomotive is used. Catenary and working state will directly affect the quality of the electrified railway transport capacity.

Catenary inspection technology is one of the key to the construction of high-speed railway. With the continuous acceleration of railway requirement for electrification of catenary will be higher. Will be more uncertain factors, also to test equipment requirement will be higher. Therefore, continuously improve the level of detection technology and equipment to guarantee the good condition, electrification of catenary to ensure smooth electrified railway transport.

\section{2) Threshold detection system}

Threshold detection system is used to detect the metro building line [6]. Then building outline data based on the top rail and tunnel cross section image can be output. And then the limit position of threshold needs to be got to provide accurate information for maintenance work [7].

Non-contact measurement methods, Laser radar detection, random vibration detection, data fusion, data mining, wavelet analysis, fuzzy recognition, and other advanced technology will be used. Through the application of coordinate transformation and related detection principle, holographic imaging can be measured for the equipment inside the tunnel. Then we can realize $3 \mathrm{D}$ image rotation and data overrun warning through special software package.

\section{3) Integrated system}

Integrated system is responsible for providing public signal, the whole system operation and management of the industrial Ethernet, and other functions for the above three detection system. The public signs provided include distance information provided by the encoder and RFID, timing signals and etc. The above three detection systems have their hardware, software, can work independently.

\section{Conclusion}

The urban subway comprehensive detection system is a complex system, which is the collection of advanced sensor technology, computer technology, network technology, data analysis, image analysis and processing technology, artificial intelligence technology and so on.

It is not realistic that using the integrated checkout automobile for high-speed rail and electric rail simply in the urban subway. It will Faced with the problems of adaptability, cost and so on.

So it is quite urgent to analysis and research the integrated detection technology that is suitable for urban subway, to research, develop and improve urban subway integrated checkout automobile.

Urban subway comprehensive detection system is a complex system which is the collection of advanced sensor technology, computer technology, network technology, data analysis technology, image analysis and processing technology, artificial intelligence technology. In order to ensure the operation safety of subway, based on the existing detection technology in high-speed rail and electric railway, we absorb new technologies in time. And comprehensive detection technology standards which are suitable for urban subway are studied according to the characteristics of subway [8]. It is very urgent to develop an urban subway integrated check-out car which has the characteristics of high reliability, compact structure and convenient operation maintenance. At the same time it has the technical conditions and good market for developing.

\section{References}

1. W. Hou. High-speed comprehensive detection train of NO.0. Beijing. China Railway Press, (2006)

2. W. Wang, Q. He. Dynamics of high-speed rail vehicle system. Advances in mechanics, 25(1):134143 (1995)

3. Ministry of railways of the People's Republic of China. Railway technology management discipline. Beijing: China Railway Press, (2010)

4. Y. Shen, L. Li, Y. Ruan. Mobile mapping technology by vehicle-borne lidar. Infrared and Laser Engineering, 38(3):437-440 (2009) 
5. W. Wang, S. Gu, C. Yang. High-speed comprehensive detection train. Railway technology innovation, (1):12-15 (2012)

6. Q. Fang, D. Zhang, L.N.Y. Wong, Shallow tunnelling method (STM) for subway station construction in soft ground. Tunnelling \& Underground Space Technology, 29(3):10-30 (2012)
7. W. Chen, Z. Long, J. Li, Research on Real-time Dynamic Monitoring of Subway Construction. Chinese Journal of Underground Space \& Engineering, (2013)

8. J.F. Hou, J. Chen, R.L. Wang, Construction Monitoring Technology Analysis for Railway Tunnel. Applied Mechanics \& Materials, 580-583:1342-1346 (2014) 